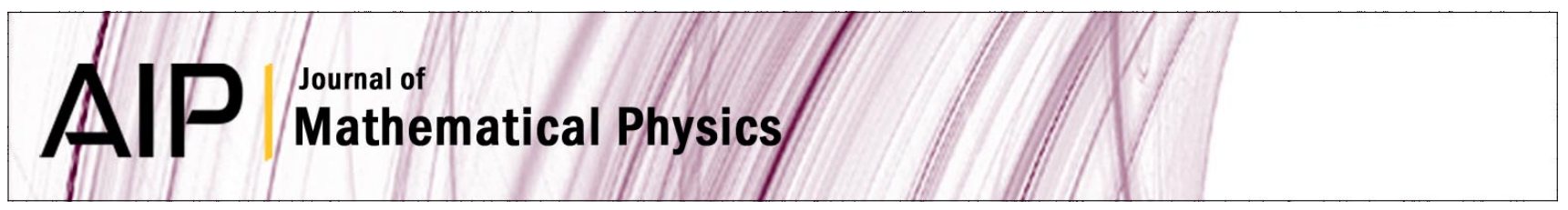

\title{
Special singularity function for continuous part of the spectral data in the associated eigenvalue problem for nonlinear equations
}

V. O. Vakhnenko and E. J. Parkes

Citation: J. Math. Phys. 53, 063504 (2012); doi: 10.1063/1.4726168

View online: http://dx.doi.org/10.1063/1.4726168

View Table of Contents: http://jmp.aip.org/resource/1/JMAPAQ/v53/i6

Published by the American Institute of Physics.

\section{Related Articles}

Soliton-like solutions to the ordinary Schrödinger equation within standard quantum mechanics J. Math. Phys. 53, 052102 (2012)

Integrability of nonlinear wave equations and solvability of their initial value problem J. Math. Phys. 53, 043701 (2012)

Spontaneous soliton generation in the higher order Korteweg-de Vries equations on the half-line Chaos 22, 013138 (2012)

The quasi-periodic solutions of mixed KdV equations J. Math. Phys. 53, 033508 (2012)

Soliton interactions in some semidiscrete integrable systems J. Math. Phys. 53, 033507 (2012)

\section{Additional information on J. Math. Phys.}

Journal Homepage: http://jmp.aip.org/

Journal Information: http://jmp.aip.org/about/about_the_journal

Top downloads: http://jmp.aip.org/features/most_downloaded

Information for Authors: http://jmp.aip.org/authors

\section{ADVERTISEMENT}

The most comprehensive support for physics in any mathematical software package World-leading tools for performing calculations in theoretical physics

The Essential Tool for Mathematics and Modeling

www.maplesoft.com/physics
Your work in Maple matches how you would write the problems and solutions by hand

State-of-the-art environment for algebraic computations in physics

The only system with the ability to handle a wide range of physics computations as well as pencil-and-paper style input and textbook-quality display of results

- Access to Maple's full mathematical power, programming language, visualization routines, and document creation tools 


\title{
Special singularity function for continuous part of the spectral data in the associated eigenvalue problem for nonlinear equations
}

\author{
V. O. Vakhnenko ${ }^{1, a)}$ and E. J. Parkes ${ }^{2, b)}$ \\ ${ }^{1}$ Institute of Geophysics, National Academy of Sciences of Ukraine, 01054 Kyï, Ukraine \\ ${ }^{2}$ Department of Mathematics and Statistics, University of Strathclyde, Glasgow G1 1XH, \\ United Kingdom
}

(Received 3 September 2011; accepted 18 May 2012; published online 11 June 2012)

\begin{abstract}
The procedure for finding the solutions of the Vakhnenko-Parkes equation by means of the inverse scattering method is described. The continuous spectrum is taken into account in the associated eigenvalue problem. The suggested special form of the singularity function for continuous part of the spectral data gives rise to the multimode solutions. The sufficient conditions are proved in order that these solutions become real functions. The interaction of the $N$ periodic waves is studied. The procedure is illustrated by considering a number of examples. (ㅇ 2012 American Institute of Physics. [http://dx.doi.org/10.1063/1.4726168]
\end{abstract}

\section{INTRODUCTION}

Various physical phenomena in engineering and physics may be described by nonlinear evolution equations. Looking for exact solutions to completely integrable equations is a difficult task. In recent years, a few methods for obtaining the exact solutions of nonlinear evolution equations have been suggested. One of the fundamental direct methods is undoubtedly the Hirota bilinear method ${ }^{1,2}$ which possesses significant features that make it practical for the determination of multiple-soliton solutions. However, the direct methods can be applied only for finding the solitary wave solutions or the traveling-wave solutions. In this sense, the inverse scattering method is the most appropriate way of tackling the initial value problem although its employment is a fairly difficult procedure. ${ }^{3-5}$

In this paper, we will consider the nonlinear evolution equation

$$
W_{X X T}+\left(1+W_{T}\right) W_{X}=0 .
$$

This equation arises from the Vakhnenko equation ${ }^{2,6-8}$

$$
\frac{\partial}{\partial x}\left(\frac{\partial}{\partial t}+u \frac{\partial}{\partial x}\right) u+u=0
$$

through the transformation 9,10

$$
\begin{aligned}
& u(x, t)=U(X, T)=W_{X}(X, T), \\
& x=x_{0}+T+W(X, T), \\
& t=X .
\end{aligned}
$$

The corresponding governing equation for $U$, namely

$$
U U_{X X T}-U_{X} U_{X T}+U^{2} U_{T}=0
$$

is given in Ref. 9.

\footnotetext{
a)E-mail: vakhnenko@ukr.net.

b)E-mail: e.j.parkes@strath.ac.uk.
} 
Equations (1), (2), and (4) arose as a result of describing high-frequency perturbations in a relaxing medium. ${ }^{8}$ Following the papers, ${ }^{11-13}$ hereafter (1) (or equivalently (4)) is referred to as the Vakhnenko-Parkes equation (VPE).

Recently the Hirota method ${ }^{2,9,10}$ as well as the inverse scattering method ${ }^{14}$ have been applied to obtain the exact $N$-soliton solutions of the VPE. In this paper, we use the inverse scattering transform method to study the periodic solutions of the VPE (1) associated with the continuous part of the spectral data.

In Sec. II, we formulate the spectral problem for the VPE by adapting the results given by Caudrey ${ }^{15}$ and by Kaup. ${ }^{16}$ In Sec. III, we find the solutions corresponding to the continuous part of the spectral data. In Sec. IV, we find the real $\mathrm{N}$-mode solutions for $N=1,2,3,4$. Our results are summarized in Sec. V.

\section{THE SPECTRAL PROBLEM FOR THE VPE}

In order to use the inverse scattering method, one first has to formulate the associated eigenvalue problem. In Ref. 14, it is shown that the pair of equations

$$
\begin{gathered}
\psi_{X X X}+W_{X} \psi_{X}-\lambda \psi=0, \\
3 \psi_{X T}+\left(W_{T}+1\right) \psi=0
\end{gathered}
$$

is associated with the VPE (1) considered here. Note that the inverse scattering transform problem is related to a spectral equation of third order (5). The inverse problem for third-order spectral equations has been considered by Caudrey ${ }^{15}$ and Kaup. ${ }^{16}$ We adapt the results obtained by these authors to the present problem and describe a procedure for using the inverse scattering transform method to find the solutions of the VPE that are associated with the continuous part of the spectral data.

We use the general theory of the inverse scattering problem for $N$ spectral equations which has been developed by Caudrey in Ref. 15. According to Ref. 15 the spectral Eq. (5) can be rewritten in the form

$$
\frac{\partial}{\partial X} \boldsymbol{\psi}=[\mathbf{A}(\zeta)+\mathbf{B}(X, \zeta)] \cdot \boldsymbol{\psi}
$$

with

$$
\boldsymbol{\psi}=\left(\begin{array}{c}
\psi \\
\psi_{X} \\
\psi_{X X}
\end{array}\right), \mathbf{A}=\left(\begin{array}{ccc}
0 & 1 & 0 \\
0 & 0 & 1 \\
\lambda & 0 & 0
\end{array}\right), \mathbf{B}=\left(\begin{array}{ccc}
0 & 0 & 0 \\
0 & 0 & 0 \\
0 & -W_{X} & 0
\end{array}\right)
$$

The matrix $\mathbf{A}$ has the eigenvalues $\lambda_{j}(\zeta)$ and left- and right-eigenvectors $\tilde{\boldsymbol{v}}_{j}(\zeta)$ and $\boldsymbol{v}_{j}(\zeta)$, respectively $(j=1,2,3)$. In the case considered here we define

$$
\begin{aligned}
& \lambda_{j}(\zeta)=\omega_{j} \zeta, \quad \lambda_{j}^{3}(\zeta)=\lambda, \\
& \boldsymbol{v}_{j}(\zeta)=\left(\begin{array}{c}
1 \\
\lambda_{j} \\
\lambda_{j}^{2}
\end{array}\right), \quad \tilde{\boldsymbol{v}}_{j}(\zeta)=\left(\begin{array}{lll}
\lambda_{j}^{2} \lambda_{j} & 1
\end{array}\right),
\end{aligned}
$$

where $\omega_{j}=e^{2 \pi \mathrm{i}(j-1) / 3}$ are the cube roots of 1 .

The solution of the linear Eq. (5), or equivalently Eq. (7), has been obtained by Caudrey ${ }^{15}$ in terms of Jost functions $\phi_{j}(X, \zeta)$ which have the asymptotic behaviour

$$
\boldsymbol{\Phi}_{j}(X, \zeta)=\exp \left\{-\lambda_{j}(\zeta) X\right\} \boldsymbol{\phi}_{j}(X, \zeta) \rightarrow \boldsymbol{v}_{j}(\zeta)
$$

as $X \rightarrow-\infty$.

Here $T$ is regarded as a parameter; the $T$-evolution of the scattering data will be taken into account later. The solution of the direct problem (7) is given by the equation system (4.5) in Ref. 15. Since there is a set of symmetry properties $\phi_{1}\left(X, \zeta / \omega_{1}\right)=\phi_{2}\left(X, \zeta / \omega_{2}\right)=\phi_{3}\left(X, \zeta / \omega_{3}\right)($ see $(6.14)$ and (6.15) in Ref. 15, for example) for Jost functions $\phi_{j}(X, \zeta)$, we need only consider the element 


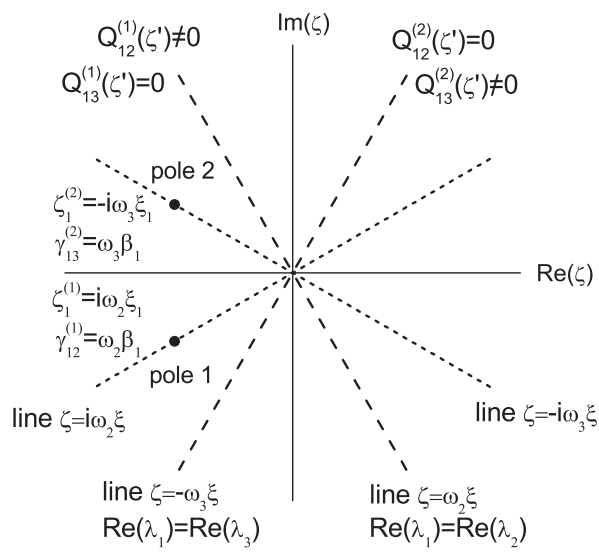

FIG. 1. The regular regions for Jost functions $\phi_{1}(X, \zeta)$ in the complex $\zeta$-plane. The dashed lines with singularity functions $Q_{1 j}\left(\zeta^{\prime}\right)$ determine the boundaries between regular regions. The dotted lines are the lines where the poles appear.

$\phi_{1}(X, \zeta)$ (as well as $\Phi_{1}(X, \zeta)$ ). In the general case, it is necessary to take into account both the bound state spectrum and the continuous spectrum. According to the relation (6.20) in Ref. 15, the solution of (7) is as follows:

$$
\begin{aligned}
\Phi_{1}(X, \zeta)= & 1-\sum_{k=1}^{K} \sum_{j=2}^{3} \gamma_{1 j}^{(k)} \frac{\exp \left\{\left[\lambda_{j}\left(\zeta_{1}^{(k)}\right)-\lambda_{1}\left(\zeta_{1}^{(k)}\right)\right] X\right\}}{\lambda_{1}\left(\zeta_{1}^{(k)}\right)-\lambda_{1}(\zeta)} \Phi_{1}\left(X, \omega_{j} \zeta_{1}^{(k)}\right) \\
& +\frac{1}{2 \pi \mathrm{i}} \int \sum_{j=2}^{3} Q_{1 j}\left(\zeta^{\prime}\right) \frac{\exp \left\{\left[\lambda_{j}\left(\zeta^{\prime}\right)-\lambda_{1}\left(\zeta^{\prime}\right)\right] X\right\}}{\zeta^{\prime}-\zeta} \Phi_{1}^{ \pm}\left(X, \omega_{j} \zeta^{\prime}\right) d \zeta^{\prime}
\end{aligned}
$$

Equation (11) contains the spectral data, namely $K$ poles with the quantities $\gamma_{1 j}^{(k)}$ for the bound state spectrum as well as the functions $Q_{1 j}\left(\zeta^{\prime}\right)$ given along all the boundaries of regular regions for the continuous spectrum. The boundaries between regions, where the Jost function $\phi_{1}(X, \zeta)$ is regular, appear at $\operatorname{Re}\left(\lambda_{1}\left(\zeta^{\prime}\right)-\lambda_{j}\left(\zeta^{\prime}\right)\right)=0$ over all $j \neq 1$ (Ref. 15) (see Fig. 1). The singularities on boundaries of these regions within the complex $\zeta$-plane are taken into account by the third term in the relation (11). The integral in (11) is along all the boundaries (see the dashed lines in Fig. 1).

The bound state spectrum is associated with soliton solutions; in this case $Q_{1 j}(\zeta) \equiv 0$ in (11). The procedure for finding the exact $N$-soliton solution of the VPE via the inverse scattering method is described in Ref. 14. In Sec. III, we study the solutions of the VPE which follow from the continuous part of the spectral data.

\section{THE SOLUTIONS ASSOCIATED WITH THE CONTINUOUS PART OF THE SPECTRAL DATA}

Now we consider only the continuous spectrum of the associated eigenvalue problem, i.e., we assume that at least some of the functions $Q_{1 j}\left(\zeta^{\prime}\right)$ are nonzero, while $\gamma_{1 j}^{(k)} \equiv 0$ in Eq. (11). At each fixed $j \neq 1$ the functions $Q_{1 j}\left(\zeta^{\prime}\right)$ characterize the singularity of $\Phi_{1}(X, \zeta)$. This singularity can appear only on boundaries between the regular regions on the $\zeta$-plane. The condition $\operatorname{Re}\left(\lambda_{1}\left(\zeta^{\prime}\right)-\lambda_{j}\left(\zeta^{\prime}\right)\right)$ $=0$ determines these boundaries. ${ }^{15}$ According to Ref. 15, we find that for $\Phi_{1}(X, \zeta)$ the complex $\zeta$-plane is divided into four regions by two lines:

$$
\begin{aligned}
& \text { (i) } \zeta=\omega_{2} \xi, \quad \text { where } Q_{12}^{(1)} \neq 0, \quad Q_{13}^{(1)} \equiv 0, \\
& \text { (ii) } \zeta=-\omega_{3} \xi, \quad \text { where } Q_{12}^{(2)} \equiv 0, \quad Q_{13}^{(2)} \neq 0,
\end{aligned}
$$

where $\xi$ is real (see Fig. 1). Analysis shows that the direction of the integration in (11) is such that $\xi$ sweeps from $-\infty$ to $+\infty$. 
Let us consider the singularity functions $Q_{1 j}\left(\zeta^{\prime}\right)$ on the boundaries, on which the Jost function $\boldsymbol{\phi}_{1}(X, \zeta)$ is singular, in the form $(n=1,2, \ldots, N)$ on the line $\zeta^{\prime}=\omega_{2} \xi$,

$$
\begin{aligned}
& Q_{12}^{(1)}\left(\zeta^{\prime}\right)=-2 \pi \mathrm{i} \sum_{n=1}^{N} q_{12}^{(2 n-1)} \delta\left(\zeta^{\prime}-\zeta_{1}^{\prime}\right), \\
& Q_{13}^{(1)}\left(\zeta^{\prime}\right)=-2 \pi \mathrm{i} \sum_{n=1}^{N} q_{13}^{(2 n-1)} \delta\left(\zeta^{\prime}-\zeta_{1}^{\prime}\right) \equiv 0,
\end{aligned}
$$

and on the line $\zeta^{\prime}=-\omega_{3} \xi$,

$$
\begin{aligned}
& Q_{12}^{(2)}\left(\zeta^{\prime}\right)=-2 \pi \mathrm{i} \sum_{n=1}^{N} q_{12}^{(2 n)} \delta\left(\zeta^{\prime}-\zeta_{2}^{\prime}\right) \equiv 0, \\
& Q_{13}^{(2)}\left(\zeta^{\prime}\right)=-2 \pi \mathrm{i} \sum_{n=1}^{N} q_{13}^{(2 n)} \delta\left(\zeta^{\prime}-\zeta_{2}^{\prime}\right) .
\end{aligned}
$$

For the singularity functions (13) and (14), the relationship (11) is reduced to the form $(M=2 N)$,

$$
\begin{aligned}
\Phi_{1}(X, \zeta)=1- & \sum_{m=1}^{M} \sum_{j=2}^{3} q_{1 j}^{(m)} \frac{\exp \left\{\left[\lambda_{j}\left(\zeta_{m}^{\prime}\right)-\lambda_{1}\left(\zeta_{m}^{\prime}\right)\right] X\right\}}{\zeta_{m}^{\prime}-\zeta} \\
& \times \Phi_{1}\left(X, \omega_{j} \zeta_{m}^{\prime}\right) .
\end{aligned}
$$

As follows from the relationship (15) and the formula

$$
\begin{aligned}
\phi_{1 X}(X, \zeta)= & \frac{\mathrm{i}}{\sqrt{3}}\left[\phi_{1 X}\left(X,-\omega_{2} \zeta\right) \phi_{1}\left(X,-\omega_{3} \zeta\right)\right. \\
& \left.-\phi_{1 X}\left(X,-\omega_{3} \zeta\right) \phi_{1}\left(X,-\omega_{2} \zeta\right)\right]
\end{aligned}
$$

given in Ref. 14, for example, the singularities in the form (13) and (14) appear in pairs $\zeta_{2 n-1}^{\prime}=\omega_{2} \xi_{n}$, $\zeta_{2 n}^{\prime}=-\omega_{3} \xi_{n}$. From (16), considering the limits $\zeta \rightarrow \zeta_{m}^{\prime}$ and $X \rightarrow-\infty$, it also follows immediately that

$$
q_{12}^{(2 n-1)} \omega_{2}=q_{13}^{(2 n)}, \quad \text { with } \quad n=1,2, \ldots, N
$$

We call attention to the fact that, at the special choice of the singularity function $Q_{1 j}\left(\zeta^{\prime}\right)$ for continuous part of the spectral data as in (13) and (14), the second term on the right-hand side of the relation (15) is similar in mathematical structure to the second term in relation (5.5) from Ref. 14. Indeed, the formal substitutions $\xi_{m}=\mathrm{i} \xi_{m}, q_{1 j}^{(m)}=\gamma_{1 j}^{(m)}$ transform the second term in (15) into the second term in (5.5) from Ref. 14. Since there is this transformation, we can apply the procedure developed for solving the $\mathrm{N}$-soliton interaction to obtain the solutions connected with the continuous part of the spectral data for the associated eigenvalue problem. ${ }^{14,15}$ According to Ref. 14 (see Eqs. (5.11) - (5.15) therein), we can find $\Phi_{1}(X, \zeta)$ and can connect $\Phi_{1}(X, \zeta)$ with the solution $W(X)$, by expanding $\Phi_{1}(X, \zeta)$ as an asymptotic series in $\lambda_{1}^{-1}(\zeta)$ (see Eq. (5.11) in Ref. 14) as follows:

$$
\Phi_{1}(X, \zeta)=1-\frac{1}{3 \lambda_{1}(\zeta)}[W(X)-W(-\infty)]+O\left(\lambda_{1}^{-2}(\zeta)\right)
$$

On the other hand, by defining

$$
\Psi_{m}(X)=\sum_{j=2}^{3} q_{1 j}^{(m)} \exp \left\{\lambda_{j}\left(\zeta_{m}^{\prime}\right) X\right\} \Phi_{1}\left(X, \omega_{j} \zeta_{m}^{\prime}\right),
$$

we may rewrite the relationship (15) as

$$
\Phi_{1}(X, \zeta)=1-\sum_{m=1}^{M} \frac{\exp \left\{-\lambda_{1}\left(\zeta_{m}^{\prime}\right) X\right\}}{\zeta_{m}^{\prime}-\zeta} \Psi_{m}(X)
$$


From (18) and (20), the following key relationship can be derived (see also Eq. (6.38) in Ref. 15):

$$
\begin{aligned}
W(X)-W(-\infty) & =-3 \sum_{m=1}^{M} \exp \left\{-\lambda_{1}\left(\zeta_{m}^{\prime}\right) X\right\} \Psi_{m}(X) \\
& =3 \frac{\partial}{\partial X} \ln (\operatorname{det} M(X))
\end{aligned}
$$

Here $M(X)$ is the $2 N \times 2 N$ matrix given by elements

$$
M_{m l}(X)=\delta_{m l}-\sum_{j=2}^{3} q_{1 j}^{(m)} \frac{\exp \left\{\left[\lambda_{j}\left(\zeta_{m}^{\prime}\right)-\lambda_{1}\left(\zeta_{l}^{\prime}\right)\right] X\right\}}{\zeta_{l}^{\prime}-\omega_{j} \zeta_{m}^{\prime}}
$$

Now let us consider the $T$-evolution of the spectral data. By analyzing the solution of Eq. (6) when $X \rightarrow-\infty$, we find that $\phi_{i}(X, T, \zeta)=\exp \left[-\left(3 \lambda_{i}(\zeta)\right)^{-1} T\right] \phi_{i}(X, 0, \zeta)$. Hence the $T$-evolution of the scattering data is given by the relationships (with $m=1,2, \ldots, M$ )

$$
\begin{aligned}
q_{i j}^{(m)}(T)= & q_{i j}^{(m)}(0) \exp \left\{\left[-\left(3 \lambda_{j}\left(\zeta_{m}^{\prime}\right)^{-1}\right.\right.\right. \\
& \left.\left.+\left(3 \lambda_{1}\left(\zeta_{m}^{\prime}\right)\right)^{-1}\right] T\right\} \\
\lambda_{j}(T)= & \lambda_{j}(0)
\end{aligned}
$$

Consequently, the final result for the solution of the VPE, when we consider the spectral data from the continuous spectrum, as well as taking into account their $T$-evolution, is as follows:

$$
W(X, T)=3 \frac{\partial}{\partial X} \ln (\operatorname{det} M(X, T))+\text { const. }
$$

The $2 N \times 2 N$ matrix $M(X, T)$ is defined as follows:

$$
M_{m l}(X, T)=\delta_{m l}-\sum_{j=2}^{3} q_{1 j}^{(m)}(0) \frac{\exp \left\{\left[-\left(3 \lambda_{j}\left(\zeta_{m}^{\prime}\right)^{-1}+\left(3 \lambda_{1}\left(\zeta_{m}^{\prime}\right)\right)^{-1}\right] T+\left[\lambda_{j}\left(\zeta_{m}^{\prime}\right)-\lambda_{1}\left(\zeta_{l}^{\prime}\right)\right] X\right\}\right.}{\zeta_{l}^{\prime}-\omega_{j} \zeta_{m}^{\prime}}
$$

with the relations $(n=1,2, \ldots, N)$

$$
\begin{array}{llll}
\lambda_{1}\left(\zeta_{2 n-1}^{\prime}\right)=\omega_{2} \xi_{2 n-1}, & \lambda_{2}\left(\zeta_{2 n-1}^{\prime}\right)=\omega_{3} \xi_{2 n-1}, & q_{12}^{(2 n-1)}=\omega_{2} \beta_{2 n-1}, & q_{13}^{(2 n-1)}=0, \\
\lambda_{1}\left(\zeta_{2 n}^{\prime}\right)=-\omega_{3} \xi_{2 n-1}, & \lambda_{3}\left(\zeta_{2 n}^{\prime}\right)=-\omega_{2} \xi_{2 n-1}, & q_{12}^{(2 n)}=0, & q_{13}^{(2 n)}=\omega_{3} \beta_{2 n-1} .
\end{array}
$$

As will be clear from the examples in Sec. IV, the solution (24) and (25) include $N$ frequencies from the continuous part of the spectral data. For this reason, the solution (24) and (25) will be referred to as the $N$-mode solution of the VPE. Evidently, these discrete modes emanate from the special choice (13) and (14) of the singularity functions $Q_{1 j}\left(\zeta^{\prime}\right)$ for continuous part of the spectral data.

For the solution (24) and (25), there are $N$ arbitrary constants $\xi_{n}$ and $N$ arbitrary constants $\beta_{n}$. The constants $\xi_{n}$ are real, while the constants $\beta_{n}$, in the general case, are complex. The solution (24) obtained through the matrix (25) is, in general, a complex function. Consequently, there is a problem in selecting the real solutions from the complex solutions. It turns out that we can obtain the real solutions by means of restriction of arbitrariness in the choice of the constants $\beta_{i}$. For the $N$-mode solution, we have succeeded in finding these restrictions.

\section{REAL PERIODIC SOLUTIONS OF THE VPE}

This research has culminated in finding the real $N$-mode solution. For convenience, we consider the solutions for $N=1,2,3,4$. For $N \geq 5$, all formulas can be easily obtained beginning with these examples. 
1. In order to obtain the one-mode solution of the VPE (1), we need first to calculate the $2 \times 2$ matrix $M(X, T)$ according to (25). For the matrix elements $M_{k l}(X, T)$, we have

$$
\begin{aligned}
& M_{11}=1-\frac{\mathrm{i} \omega_{2} \beta_{1}}{\sqrt{3} \xi_{1}} \exp \left[-\mathrm{i} \sqrt{3} \xi_{1} X+\left(\mathrm{i} \sqrt{3} \xi_{1}\right)^{-1} T\right], \\
& M_{12}=-\frac{\omega_{3} \beta_{1}}{2 \xi_{1}} \exp \left[2 \omega_{3} \xi_{1} X+\left(\mathrm{i} \sqrt{3} \xi_{1}\right)^{-1} T\right], \\
& M_{21}=\frac{\omega_{2} \beta_{1}}{2 \xi_{1}} \exp \left[-2 \omega_{2} \xi_{1} X+\left(\mathrm{i} \sqrt{3} \xi_{1}\right)^{-1} T\right], \\
& M_{22}=1-\frac{\mathrm{i} \omega_{3} \beta_{1}}{\sqrt{3} \xi_{1}} \exp \left[-\mathrm{i} \sqrt{3} \xi_{1} X+\left(\mathrm{i} \sqrt{3} \xi_{1}\right)^{-1} T\right],
\end{aligned}
$$

so that its determinant is

$$
\operatorname{det} M=\left[1+c_{1} \exp \left(-\mathrm{i} \sqrt{3} \xi_{1} X+\left(\mathrm{i} \sqrt{3} \xi_{1}\right)^{-1} T\right)\right]^{2},
$$

where $c_{1}=-\frac{\mathrm{i} \beta_{1}}{2 \sqrt{3} \xi_{1}}$.

As has already been noted, the singularity functions in the form (13) and (14) give rise to a single frequency for the continuous part of the spectral data. Hence, once the expression (28) has been substituted into the key formula (24), (24) must provide us with the one-mode solution.

The condition that $W_{X}$ is real requires a restriction on the constant $\beta_{1}$ (if the constant $\xi_{1}$ is arbitrary, but real). We have succeeded in obtaining this restriction (see Appendix), namely that the constant $c_{1}$, which in general is the complex-valued one $c_{1}=\left|c_{1}\right| \exp \left(\mathrm{i} \chi_{1}\right)$, should possess unit modulus $\left|c_{1}\right|=1$, while the arbitrary real constant $\chi_{1}$ defines an initial shift of solution $X_{1}=\chi_{1} /\left(\sqrt{3} \xi_{1}\right)$ so that

$$
\operatorname{det} M=\left[1+\exp \left(-\mathrm{i} \sqrt{3} \xi_{1}\left(X-X_{1}\right)+\frac{T}{\mathrm{i} \sqrt{3} \xi_{1}}\right)\right]^{2} .
$$

The final result for one mode of the continuous spectrum is the solution (24) with (29), namely,

$$
W=-3 \sqrt{3} \xi_{1} \tan \left(\frac{\sqrt{3}}{2} \xi_{1}\left(X-X_{1}\right)+\frac{T}{2 \sqrt{3} \xi_{1}}\right)+\text { const. }
$$

The corresponding solution for $U=W_{X}$ (with $U$ governed by (4)) was obtained recently by other methods, for example, by the sine-cosine method, ${ }^{17}$ the $\left(G^{\prime} / G\right)$-expansion method, ${ }^{13}$ and the extended tanh-function method. ${ }^{17-19}$ However, only the approach developed here and the solution in the form (24) and (25) enable us to study the interaction the periodic $N$-mode waves.

2. Let us consider the two-mode solution of the VPE. In this case $M(X, T)$ is a $4 \times 4$ matrix. We will not give the explicit form of this matrix here, but we find its determinant

$$
\operatorname{det} M(X, T)=\left(1+q_{1}+q_{2}+b_{12} q_{1} q_{2}\right)^{2},
$$

where

$$
\begin{gathered}
q_{i}=c_{i} \exp \left[-\mathrm{i} \sqrt{3} \xi_{i} X+\left(\mathrm{i} \sqrt{3} \xi_{i}\right)^{-1} T\right], \quad c_{i}=-\frac{\mathrm{i} \beta_{i}}{2 \sqrt{3} \xi_{i}}, \\
b_{12}=\left(\frac{\xi_{2}-\xi_{1}}{\xi_{2}+\xi_{1}}\right)^{2} \frac{\xi_{1}^{2}+\xi_{2}^{2}-\xi_{1} \xi_{2}}{\xi_{1}^{2}+\xi_{2}^{2}+\xi_{1} \xi_{2}}, \quad b_{12} \geq 0 .
\end{gathered}
$$

In Appendix, the restrictions on the constants $c_{i}=\left|c_{i}\right| \exp \left(\mathrm{i} \chi_{i}\right)$ for real solutions are found. The real constants $\chi_{i}$ define the initial shifts of solutions $X_{i}=\chi_{i} /\left(\sqrt{3} \xi_{i}\right)$. The analysis in considerable detail shows (see Appendix) that the relations $\left|c_{1}\right|=\left|c_{2}\right|=1 / \sqrt{b_{12}}$ are sufficient conditions in order that $W$ may become real. Consequently, the real solution describing the interaction of two periodic waves for the VPE is defined by the key relationship (24), where

$$
\operatorname{det} M(X, T)=\left(1+\frac{1}{\sqrt{b_{12}}} q_{1}+\frac{1}{\sqrt{b_{12}}} q_{2}+q_{1} q_{2}\right)^{2}
$$


and $b_{12}$ is as in (32), while $q_{i}$ should contain the phase shifts $X_{i}=\chi_{i} /\left(\sqrt{3} \xi_{i}\right)$ as in (34), namely

$$
q_{i}=\exp \left[-\mathrm{i} \sqrt{3} \xi_{i}\left(X-X_{i}\right)+\left(\mathrm{i} \sqrt{3} \xi_{i}\right)^{-1} T\right] .
$$

3. For $N=3$ in relationship obtained from (25)

$$
\begin{aligned}
\operatorname{det} M(X, T)= & \left(1+c_{1} q_{1}+c_{2} q_{2}+c_{3} q_{3}+c_{1} c_{2} b_{12} q_{1} q_{2}\right. \\
& +c_{1} c_{3} b_{13} q_{1} q_{3}+c_{2} c_{3} b_{23} q_{2} q_{3} \\
& \left.+c_{1} c_{2} c_{3} b_{12} b_{13} b_{23} q_{1} q_{2} q_{3}\right)^{2}
\end{aligned}
$$

with $q_{i}, c_{i}$ as in (32) and

$$
b_{i j}=\left(\frac{\xi_{j}-\xi_{i}}{\xi_{j}+\xi_{i}}\right)^{2} \frac{\xi_{i}^{2}+\xi_{j}^{2}-\xi_{i} \xi_{j}}{\xi_{i}^{2}+\xi_{j}^{2}+\xi_{i} \xi_{j}}, \quad b_{i j}=b_{j i},
$$

we write $c_{i}=\left|c_{i}\right| \exp \left(\mathrm{i} \chi_{i}\right)$, then the arguments $\chi_{i}$ determine the initial phase shifts of mode $X_{i}$ $=\chi_{i} /\left(\sqrt{3} \xi_{i}\right)$. As is proved in Appendix, the conditions on the constants $c_{1}$ (or the same on $\beta_{i}$ ) are

$$
\left|c_{1}\right|=1 / \sqrt{b_{12} b_{13}},\left|c_{2}\right|=1 / \sqrt{b_{12} b_{23}},\left|c_{3}\right|=1 / \sqrt{b_{13} b_{23}} .
$$

Hence, the three-mode solution is the relation (24) with

$$
\begin{aligned}
\operatorname{det} M= & {\left[1+\frac{1}{\sqrt{b_{12} b_{13}}}\left(q_{1}+q_{2} q_{3}\right)+\frac{1}{\sqrt{b_{12} b_{23}}}\left(q_{2}+q_{1} q_{3}\right)\right.} \\
& \left.+\frac{1}{\sqrt{b_{13} b_{23}}}\left(q_{3}+q_{1} q_{2}\right)+q_{1} q_{2} q_{3}\right]^{2} .
\end{aligned}
$$

Here the phase shifts $X_{i}$ are taken into account in $q_{i}$ by way of (34).

4. For $N=4$ the restrictions are as follows (see Appendix):

$$
\left|c_{i}\right|=\prod_{j \neq i}^{4} b_{i j}^{-\frac{1}{2}}, \quad b_{i j}=b_{j i}, \quad i=1,2,3,4 .
$$

The determinant for a real solution (24) is as follows:

$$
\begin{aligned}
\operatorname{det} M= & {\left[1+\frac{1}{\sqrt{b_{12} b_{13} b_{14}}}\left(q_{1}+q_{2} q_{3} q_{4}\right)\right.} \\
& +\frac{1}{\sqrt{b_{12} b_{23} b_{24}}}\left(q_{2}+q_{1} q_{3} q_{4}\right) \\
& +\frac{1}{\sqrt{b_{13} b_{23} b_{34}}}\left(q_{3}+q_{1} q_{2} q_{4}\right) \\
& +\frac{1}{\sqrt{b_{14} b_{24} b_{34}}}\left(q_{4}+q_{1} q_{2} q_{3}\right) \\
& +\frac{1}{\sqrt{b_{13} b_{14} b_{23} b_{24}}}\left(q_{1} q_{2}+q_{3} q_{4}\right) \\
& +\frac{1}{\sqrt{b_{12} b_{14} b_{23} b_{34}}}\left(q_{1} q_{3}+q_{2} q_{4}\right) \\
& \left.+\frac{1}{\sqrt{b_{12} b_{13} b_{24} b_{34}}}\left(q_{1} q_{4}+q_{2} q_{3}\right)+q_{1} q_{2} q_{3} q_{4}\right]^{2} .
\end{aligned}
$$

As before, the $q_{i}$ and $b_{i j}$ are defined by (34) and (36), respectively. 


\section{CONCLUSION}

We have adapted and applied the inverse scattering method to the Vakhnenko-Parkes equation in order to find the solutions that are associated with the continuous spectrum of the spectral problem. The special form of the singularity function for continuous part of the spectral data enabled us to obtain the multi-mode solutions. The sufficient conditions have been proved in order that the solutions become real functions. We have described how to define the interaction of the multi-mode periodic waves. The procedure has been illustrated by considering a number of examples.

\section{ACKNOWLEDGMENTS}

V.O.V. is grateful to K. P. Kutsevol for stimulating criticism and helpful discussions.

\section{APPENDIX: THE CONDITIONS ON THE CONSTANTS $c_{i}$ FOR REAL SOLUTIONS}

We use the case $N=4$ as an example to prove the restrictions on the constants, at which the solution $W(X, T)$ is real. We will consider the auxiliary function $f=\sqrt{\operatorname{det} M(X, T)}$ for convenience, namely

$$
\begin{aligned}
f= & 1+c_{1} q_{1}+c_{2} q_{2}+c_{3} q_{3}+c_{4} q_{4}+c_{1} c_{2} b_{12} q_{1} q_{2} \\
& +c_{1} c_{3} b_{13} q_{1} q_{3}+c_{1} c_{4} b_{14} q_{1} q_{4}+c_{2} c_{3} b_{23} q_{2} q_{3} \\
& +c_{2} c_{4} b_{24} q_{2} q_{4}+c_{3} c_{4} b_{34} q_{3} q_{4}+c_{1} c_{2} c_{3} b_{12} b_{13} b_{23} q_{1} q_{2} q_{3} \\
& +c_{1} c_{2} c_{4} b_{12} b_{14} b_{24} q_{1} q_{2} q_{4}+c_{1} c_{3} c_{4} b_{13} b_{14} b_{34} q_{1} q_{3} q_{4} \\
& +c_{2} c_{3} c_{4} b_{23} b_{24} b_{34} q_{2} q_{3} q_{4} \\
& +c_{1} c_{2} c_{3} c_{4} b_{12} b_{13} b_{14} b_{23} b_{24} b_{34} q_{1} q_{2} q_{3} q_{4} .
\end{aligned}
$$

We here redefine the values $c_{i}=\left|c_{i}\right|$, since the arguments $\chi_{i}$ can always be introduced in the variables $q_{i}=\exp \left(i \theta_{i}\right)$ with $\theta=-\sqrt{3} \xi_{i}\left(X-X_{i}\right)-\left(\sqrt{3} \xi_{i}\right)^{-1} T$.

The solution (21) then has a form

$$
W(X, T)=6 \frac{\partial}{\partial X} \ln (f)+\text { const. }
$$

The function $f$ is complex-valued, i.e.,

$$
\begin{aligned}
& f=f_{R e}+\mathrm{i} f_{I m}=|f| \exp \left(\mathrm{i} \chi_{f}\right), \quad f_{R e}=\operatorname{Re}(f), \\
& f_{I m}=\operatorname{Im}(f), \quad \tan \left(\chi_{f}\right)=f_{I m} / f_{R e},
\end{aligned}
$$

hence,

$$
W(X, T) / 6=\frac{\partial}{\partial X} \ln (|f|)+\mathrm{i} \frac{\partial \chi_{f}}{\partial X}+\text { const. }
$$

If we succeed in making $\partial^{2} \chi_{f} / \partial X^{2} \equiv 0$ by the choice of the constants $c_{i}$, then the solution $W(X, T)$ will be a real function. 
Let us write $f_{I m}$ and $f_{R e}$ in explicit forms

$$
\begin{aligned}
f_{I m}= & c_{1} \sin \left(\theta_{1}\right)+c_{2} \sin \left(\theta_{2}\right)+c_{3} \sin \left(\theta_{3}\right)+c_{4} \sin \left(\theta_{4}\right) \\
& +c_{1} c_{2} b_{12} \sin \left(\theta_{1}+\theta_{2}\right)+c_{1} c_{3} b_{13} \sin \left(\theta_{1}+\theta_{3}\right) \\
& +c_{1} c_{4} b_{14} \sin \left(\theta_{1}+\theta_{4}\right)+c_{2} c_{3} b_{23} \sin \left(\theta_{2}+\theta_{3}\right) \\
& +c_{2} c_{4} b_{24} \sin \left(\theta_{2}+\theta_{4}\right)+c_{3} c_{4} b_{34} \sin \left(\theta_{3}+\theta_{4}\right) \\
& +c_{1} c_{2} c_{3} b_{12} b_{13} b_{23} \sin \left(\theta_{1}+\theta_{2}+\theta_{3}\right) \\
& +c_{1} c_{2} c_{4} b_{12} b_{14} b_{24} \sin \left(\theta_{1}+\theta_{2}+\theta_{4}\right) \\
& +c_{1} c_{3} c_{4} b_{13} b_{14} b_{34} \sin \left(\theta_{1}+\theta_{3}+\theta_{4}\right) \\
& +c_{2} c_{3} c_{4} b_{23} b_{24} b_{34} \sin \left(\theta_{2}+\theta_{3}+\theta_{4}\right) \\
& +c_{1} c_{2} c_{3} c_{4} b_{12} b_{13} b_{14} b_{23} b_{24} b_{34} \sin \left(\theta_{1}+\theta_{2}+\theta_{3}+\theta_{4}\right) \\
& \\
f_{R e}= & +c_{1} \cos \left(\theta_{1}\right)+c_{2} \cos \left(\theta_{2}\right)+c_{3} \cos \left(\theta_{3}\right)+c_{4} \cos \left(\theta_{4}\right) \\
& +c_{1} c_{2} b_{12} \cos \left(\theta_{1}+\theta_{2}\right)+c_{1} c_{3} b_{13} \cos \left(\theta_{1}+\theta_{3}\right) \\
& +c_{1} c_{4} b_{14} \cos \left(\theta_{1}+\theta_{4}\right)+c_{2} c_{3} b_{23} \cos \left(\theta_{2}+\theta_{3}\right) \\
& +c_{2} c_{4} b_{24} \cos \left(\theta_{2}+\theta_{4}\right)+c_{3} c_{4} b_{34} \cos \left(\theta_{3}+\theta_{4}\right) \\
& +c_{1} c_{2} c_{3} b_{12} b_{13} b_{23} \cos \left(\theta_{1}+\theta_{2}+\theta_{3}\right) \\
& +c_{1} c_{2} c_{4} b_{12} b_{14} b_{24} \cos \left(\theta_{1}+\theta_{2}+\theta_{4}\right) \\
& +c_{1} c_{3} c_{4} b_{13} b_{14} b_{34} \cos \left(\theta_{1}+\theta_{3}+\theta_{4}\right) \\
& +c_{2} c_{3} c_{4} b_{23} b_{24} b_{34} \cos \left(\theta_{2}+\theta_{3}+\theta_{4}\right) \\
& +c_{1} c_{2} c_{3} c_{4} b_{12} b_{13} b_{14} b_{23} b_{24} b_{34} \cos \left(\theta_{1}+\theta_{2}+\theta_{3}+\theta_{4}\right) \\
& \\
&
\end{aligned}
$$

Now we select a factor $\sin \left(\frac{1}{2}\left(\theta_{1}+\theta_{2}+\theta_{3}+\theta_{4}\right)\right)$ from $f_{\text {Im }}$ and a factor $\cos \left(\frac{1}{2}\left(\theta_{1}+\theta_{2}+\theta_{3}+\theta_{4}\right)\right)$ from $f_{R e}$. This can be done if the following conditions are satisfied:

$$
\begin{aligned}
& c_{1}=c_{2} c_{3} c_{4} b_{23} b_{24} b_{34}, c_{2}=c_{1} c_{3} c_{4} b_{13} b_{14} b_{34}, \\
& c_{3}=c_{1} c_{2} c_{4} b_{12} b_{14} b_{24}, c_{4}=c_{1} c_{2} c_{3} b_{12} b_{13} b_{23}, \\
& c_{1} c_{2} b_{12}=c_{3} c_{4} b_{34}, c_{1} c_{3} b_{13}=c_{2} c_{4} b_{24}, c_{1} c_{4} b_{14}=c_{2} c_{3} b_{23}, \\
& c_{1} c_{2} c_{3} c_{4} b_{12} b_{13} b_{14} b_{23} b_{24} b_{34}=1 .
\end{aligned}
$$

It turns out that all these relations are valid, when

$$
\begin{array}{ll}
c_{1}=\frac{1}{\sqrt{b_{12} b_{13} b_{14}}}, & c_{2}=\frac{1}{\sqrt{b_{12} b_{23} b_{24}}}, \\
c_{3}=\frac{1}{\sqrt{b_{13} b_{23} b_{34}}}, & c_{4}=\frac{1}{\sqrt{b_{14} b_{24} b_{34}}} .
\end{array}
$$

The conditions (A8) enable us to reduce both $f_{I m}$ and $f_{R e}$ to the forms

$$
\begin{aligned}
& f_{I m}=2 g \sin \left(\frac{1}{2}\left(\theta_{1}+\theta_{2}+\theta_{3}+\theta_{4}\right)\right), \\
& f_{R e}=2 g \cos \left(\frac{1}{2}\left(\theta_{1}+\theta_{2}+\theta_{3}+\theta_{4}\right)\right),
\end{aligned}
$$


where

$$
\begin{aligned}
g= & \frac{1}{\sqrt{b_{12} b_{13} b_{14}}} \cos \left(\frac{1}{2}\left(\theta_{1}-\theta_{2}-\theta_{3}-\theta_{4}\right)\right) \\
& +\frac{1}{\sqrt{b_{12} b_{23} b_{24}}} \cos \left(\frac{1}{2}\left(\theta_{2}-\theta_{1}-\theta_{3}-\theta_{4}\right)\right) \\
& +\frac{1}{\sqrt{b_{13} b_{23} b_{34}}} \cos \left(\frac{1}{2}\left(\theta_{3}-\theta_{1}-\theta_{2}-\theta_{4}\right)\right) \\
& +\frac{1}{\sqrt{b_{14} b_{24} b_{34}}} \cos \left(\frac{1}{2}\left(\theta_{4}-\theta_{1}-\theta_{2}-\theta_{3}\right)\right) \\
& +\frac{1}{\sqrt{b_{13} b_{14} b_{23} b_{24}}} \cos \left(\frac{1}{2}\left(\theta_{1}+\theta_{2}-\theta_{3}-\theta_{4}\right)\right) \\
& +\frac{1}{\sqrt{b_{12} b_{14} b_{23} b_{34}}} \cos \left(\frac{1}{2}\left(\theta_{1}+\theta_{3}-\theta_{2}-\theta_{4}\right)\right) \\
& +\frac{1}{\sqrt{b_{12} b_{13} b_{24} b_{34}}} \cos \left(\frac{1}{2}\left(\theta_{1}+\theta_{4}-\theta_{2}-\theta_{3}\right)\right) .
\end{aligned}
$$

Now it is readily seen from (A3) that

$$
\chi_{f}=\frac{1}{2}\left(\theta_{1}+\theta_{2}+\theta_{3}+\theta_{4}\right)
$$

and as a consequence we have

$$
\frac{\partial^{2} \chi_{f}}{\partial X^{2}}=\frac{\partial^{2} \chi_{f}}{\partial X \partial T}=0
$$

Hence, as follows from (A4), the four-mode solution of the VPE can be reduced to real form with four real constants $X_{i}$ and four real constants $\xi_{i}$ (see (41)).

Without proof here we give the following conditions on the constants $c_{i}$ that ensure the real $N$-mode solution of the VPE:

$$
\left|c_{i}\right|=\prod_{j \neq i}^{N} b_{i j}^{-\frac{1}{2}}, \quad b_{i j}=b_{j i}, \quad i=1, \ldots, N,
$$

whereas the $N$ constants $\xi_{i}$ determine the values $b_{i j}$ and the $N$ constants $X_{i}$ through $\beta_{i}$ define the phase shifts of the separate modes. Note that these relations are sufficient conditions, but not necessary ones.

${ }^{1}$ R. Hirota, The Direct Method in Soliton Theory (Cambridge University Press, 2004).

${ }^{2}$ A. M. Wazwaz, Phys. Scr. 82, 065006 (2010).

${ }^{3}$ M. J. Ablowitz and H. Segur, Solitons and the Inverse Scattering Transform (SIAM, 1981).

${ }^{4}$ Bäcklund Transformations, the Inverse Scattering Method, Solitons, and Their Applications, edited by R. M. Miura (Springer, 1976).

${ }^{5}$ S. P. Novikov, S. V. Manakov, L. P. Pitaevskii, and V. E. Zakharov, Theory of Solitons: The Inverse Scattering Methods (Plenum, 1984).

${ }^{6}$ V. A. Vakhnenko, J. Phys. A 25, 4181 (1992).

${ }^{7}$ E. J. Parkes, J. Phys. A 26, 6469 (1993).

${ }^{8}$ V. O. Vakhnenko, J. Math. Phys. 40, 2011 (1999).

${ }^{9}$ V. O. Vakhnenko and E. J. Parkes, Nonlinearity 11, 1457 (1998).

${ }^{10}$ A. J. Morrison, E. J. Parkes, and V. O. Vakhnenko, Nonlinearity 12, 1427 (1999).

${ }^{11}$ M. L. Gandarias and M. S. Bruzón, "Symmetry reductions and exact solutions for the Vakhnenko equation," in Proceedings of XXI Congreso de Ecuaciones Diferenciales y Aplicacions, XI Congreso de Matimática Aplicada, Ciudad Real, 21-25 Septiembre 2009, pp. 1-6.

${ }^{12}$ P. G. Estévez, Theor. Math. Phys. 159, 762 (2009).

${ }^{13}$ R. Abazari, Comput. Fluids 39, 1957 (2010). 
${ }^{14}$ V. O. Vakhnenko and E. J. Parkes, Chaos, Solitons Fractals 13/9, 1819 (2002).

${ }^{15}$ P. J. Caudrey, Physica D 6, 51 (1982).

${ }^{16}$ D. J. Kaup, Stud. Appl. Math. 62, 189 (1980).

${ }^{17}$ E. Yusufoglu and A. Bekir, Chaos, Solitons Fractals 38, 1126 (2008).

${ }^{18}$ E. J. Parkes, Appl. Math. Comput. 217, 3575 (2010).

${ }^{19}$ E. J. Parkes, Comput. Fluids 42, 108 (2011). 